BI-TP 2005/30

\title{
The impact of QCD plasma instabilities on bottom-up thermalization
}

\author{
Dietrich Bödeker ${ }^{1}$ \\ Fakultät für Physik, Universität Bielefeld, 33615 Bielefeld, Germany
}

\begin{abstract}
QCD plasma instabilities, caused by an anisotropic momentum distributions of the particles in the plasma, are likely to play an important role in thermalization in heavy ion collisions. We consider plasmas with two different components of particles, one strongly anisotropic and one isotropic or nearly isotropic. The isotropic component does not eliminate instabilities but it decreases their growth rates. We investigate the impact of plasma instabilities on the first stage of the "bottom-up" thermalization scenario in which such a two-component plasma emerges, and find that even in the case of non-abelian saturation instabilities qualitatively change the bottom-up picture.
\end{abstract}

\footnotetext{
${ }^{1}$ e-mail: bodeker@physik.uni-bielefeld.de
} 


\section{Introduction}

It is an interesting and still open question whether in heavy ion collisions it is possible to create a medium made of quarks and gluons which is locally in thermal equilibrium, which would allow to study thermodynamics of QCD. On the theoretical side it would be desirable to have an answer to this question in a limit when one has perturbative control, which could be the case for very large collision energies.

This question can probably be divided into two. The first concerns the earliest stage of the collision when partons are "freed" from the colliding nuclei. The second is how this system subsequently evolves in time, and whether and when it approaches local thermal equilibrium. It should be possible to address the second question in perturbation theory, provided the produced partons have sufficiently large energies and are sufficiently dense so that multiple scatterings occur.

In the saturation scenario for high energy nucleus-nucleus scattering [1] the freed partons are mostly gluons with transverse momenta of order of the so called saturation scale $Q \gg \Lambda_{\mathrm{QCD}}$. The distribution function in phase space, or occupation number $f(x, \boldsymbol{p})$ of these gluons is of order $1 / g^{2}$, where $g$ is the QCD gauge coupling $\left(\alpha_{\mathrm{s}}=\right.$ $\left.g^{2} /(4 \pi)\right)$. The scale that sets the value of $g$ is expected to be $Q$, so that for large collision energies the occupation number is large. Since the typical occupation number in thermal equilibrium is of order one, this system is far from equilibrium.

It is customary to assume that $f$ does not depend on the coordinates transverse to the beam direction, which we choose as the $z$-axis, and that $f$ is invariant under rotations around $\hat{\boldsymbol{z}}$. This should be a good approximation for sufficiently large nuclei and for sufficiently central (head-on) collisions. Furthermore, it is assumed that the gluon distribution function is invariant under boosts in $z$-direction. With these assumptions $f$ only depends on $\tau=\sqrt{t^{2}-z^{2}}$, on the magnitude of the momentum perpendicular to $\hat{\boldsymbol{z}}$, and on the difference of the momentum- and coordinate-space rapidities.

After the gluons are freed around $Q \tau \sim 1$, the system expands and the occupation number of the originally produced, so called "hard" gluons drops below $1 / g^{2}$. Without production of additional gluons their number density $n_{\mathrm{h}}$ decreases like $1 / \tau$. It is not at all obvious [2, 3] that such a system thermalizes which would require that the relevant interaction rate is larger than the expansion rate $1 / \tau$. Consider for instance the rate $\Gamma$ for $2 \rightarrow 2$ large angle scattering of hard gluons for which the cross section $\sigma$ is of order $g^{4} / Q^{2}$. Taking into account Bose enhancement and using $n_{\mathrm{h}} \sim n_{\mathrm{h}, \text { initial }} /(Q \tau) \sim$ 
$Q^{3} /\left(g^{2} Q \tau\right)$ one can estimate

$$
\Gamma \sim \sigma n_{\mathrm{h}}\left(1+f_{\mathrm{h}}\right) \sim g^{2}\left(1+f_{\mathrm{h}}\right) / \tau
$$

which is always small compared to the expansion rate.

In Ref. [4 it was argued that inelastic processes play an essential role for thermalization. In the detailed thermalization scenario of Ref. [5] "soft" gluons are produced by bremsstrahlung off the hard gluons. Initially most of the soft gluons have the smallest possible energy, but then their momentum rapidly increases in multiple elastic scatterings with the hard gluons. The soft gluons thermalize among themselves, and are then heated by the hard gluons which eventually loose all their energy to the soft-gluon heat bath ("bottom-up thermalization").

While there is no minimal momentum transfer for massless gluon exchange in the vacuum, a medium usually cuts off long range interactions. For particles with nearly isotropic momentum distributions this can be accounted for by including the polarization tensor in the propagators of the exchanged gluons ${ }^{2}$. However, due to the longitudinal expansion the typical longitudinal momentum of hard gluons $p_{\mathrm{h}}^{z}$ is much smaller than the transverse momentum which for a long time remains order $Q$, so that the momentum distribution of the hard gluons is strongly anisotropic. Anisotropic distributions can cause so called plasma ${ }^{3}$ instabilities which means that some long wavelength $(|\boldsymbol{k}| \ll Q)$ gauge field modes grow exponentially if their amplitudes are sufficiently small. This is a collective phenomenon which is not visible in the kinetic equation framework of this problem used in 2, 3, 4, 5. The effect has been known for many years in plasma physics, and it has been argued [6] that QCD plasma instabilities can speed up equilibration in heavy ion collisions since they tend to make the momentum distributions more isotropic.

The possible relevance of plasma instabilities to the bottom-up scenario was realized in Ref. 7] where also the qualitative difference between QED and QCD plasma instabilities was discussed. At some point the growth of instabilities is stopped by non-linear effects. In QED this happens when the amplitude of the unstable modes has become so large that they deflect a particle's momenta by a large angle over the distance of one wavelength of an unstable mode. This corresponds to gauge field amplitudes $A \sim p / e$

\footnotetext{
${ }^{2}$ The situation is more complicated for magneto-static interactions, but at leading order these do not play a role in the scattering processes which are relevant to thermalization.

${ }^{3}$ Here "plasma" refers to a system of quarks and gluons which is not necessarily in thermal equilibrium, while sometimes the term "quark-gluon-plasma" is used only for thermalized or almost thermalized systems.
} 
where $p$ is a typical particle momentum. Then plasma instabilities have a dramatic effect, in particular they are able to isotropize the particle's momentum distribution within a very short time. In QCD gluons are self-interacting which may change the behavior of instabilities completely. The linear approximation already breaks down at much smaller amplitudes $A \sim k / g$ where $|\boldsymbol{k}| \ll|\boldsymbol{p}|$ is a characteristic wave vector of $A$, and a crucial question is whether these non-linearities stop the growth of instabilities. In Ref. [8] it was suggested that gluon self-interactions may not saturate the instabilities because the system can "abelianize" so that the unstable modes can grow until they hit the abelian saturation bound $A \lesssim p / g$. In this case the distribution of hard gluons would quickly become isotropic. It has been argued in Ref. [9] that this is sufficient for a hydrodynamic description to be applicable even if there is no local thermal equilibrium. This could solve the puzzle [10] why hydrodynamic calculations successfully describe experimental results for elliptic flow provided that they can be used from very early times $(\tau \sim 0.6 \mathrm{fm} / c)$ on, while perturbative estimates for thermalization times are substantially larger $(\tau \gtrsim 2.5 \mathrm{fm} / c)[9$. In lattice simulations with fields only depending on $t$ and $z$ abelianization was indeed observed [11, 12, but the recent $3+1$ dimensional simulations of Refs. [13, 14] indicate that instabilities are saturated by non-abelian interactions which would mean that their effect is less dramatic than suggested in Ref. 9]. Nevertheless the instabilities lead to larger infrared gluon fields than in an isotropic plasma and their role in the evolution of the system remains to be understood.

So far only the effect of the hard, strongly anisotropic gluons on the infrared modes has been considered in the literature. In the bottom-up scenario also the produced soft gluons, which have isotropic or nearly isotropic momentum distribution, contribute to the polarization tensor and can therefore have an influence on plasma instabilities as recently emphasized in Ref. [15]. In Sec. 2] we consider plasma instabilities in a system containing two components, one strongly anisotropic and one isotropic. In Sec. 3 we show how in the early stage of the bottom-up scenario plasma instabilities affect the momentum distribution of hard gluons even in the case of non-abelian saturation.

Notation: 4-vectors are denoted by lower case italics, 3-vectors by boldface. The metric is "mostly negative", $\left(g_{\mu \nu}\right)=\operatorname{diag}(1,-1,-1,-1)$. 


\section{Instabilities in two-component plasmas}

In the presence of instabilities one cannot naively resum the polarization tensor into the gluon propagator and then use it to compute scattering matrix elements entering a Boltzmann equation. Instead, one has to fully take into account the dynamics of the low momentum gauge field modes. Typically they have large occupation numbers and can be described by a classical gluon field $A_{\mu}=A_{\mu}^{a} T^{a}$, where $T^{a}$ are the $\mathrm{SU}(3)$ generators in the fundamental representation. When the occupation numbers of high momentum gluons are small compared to $1 / g^{2}$ they can be described as weakly interacting particles with a definite momentum. The dynamics of this coupled system satisfies the nonabelian Vlasov equations [16, 17]

$$
\begin{gathered}
\left(D_{\mu} F^{\mu \nu}\right)^{a}=g \int \frac{d^{3} p}{(2 \pi)^{3}} v^{\nu} f^{a}, \\
v \cdot D \hat{f}+\frac{1}{2} g v^{\mu}\left\{F_{\mu i}, \frac{\partial \hat{f}}{\partial p^{i}}\right\}=0
\end{gathered}
$$

where $D_{\mu}=\partial_{\mu}-i g\left[A_{\mu}, \cdot\right]$ is the adjoint representation covariant derivative, $F^{\mu \nu}$ is the field strength tensor and $v^{\mu} \equiv(1, \hat{\boldsymbol{p}})$. The distribution functions of high momentum gluons are encoded in $\hat{f}(x, \boldsymbol{p})$ which is a hermitian $N \times N$ matrix with $N=3$ being the number of colors and $f^{a}=\operatorname{tr}\left(T^{a} \hat{f}\right)$. For sufficiently small gauge field amplitudes one can write $\hat{f}=f \mathbb{1} / N+\delta \hat{f}$ and linearize with respect to $A$ and $\delta \hat{f}$. In order to see whether there are instabilities one can neglect the $x$-dependence of $f$ provided the growth rate and wave vectors of the unstable modes are large compared to the inverse of the time and lenght scales on which $f$ varies. The initial value problem can then be solved by spatial Fourier transformation and one-sided Fourier transformation with respect to time, $A\left(k_{0}\right) \equiv \int_{0}^{\infty} d t e^{i k_{0} t} A(t)$ where the frequency $k_{0}$ is in the upper half of the complex plane [18 $]^{4}$. By eliminating $\delta \hat{f}(k)$ one obtains an equation for $A(k)$ which takes the form

$$
\left[k^{2} g^{\mu \nu}-k^{\mu} k^{\nu}+\Pi^{\mu \nu}(k)\right] A_{\nu}(k)=\Phi^{\mu}(k)
$$

with the polarization tensor

$$
\begin{aligned}
\Pi^{\mu \nu}(k) & =2 N g^{2} \int \frac{d^{3} p}{(2 \pi)^{3}} \frac{\partial f(\boldsymbol{p})}{\partial p^{i}}\left(v^{\mu} \delta^{i \nu}-\frac{v^{\mu} v^{\nu} k^{i}}{v \cdot k}\right) \\
& =2 N g^{2} \int \frac{d^{3} p}{(2 \pi)^{3}} \frac{f(\boldsymbol{p})}{|\boldsymbol{p}|}\left(-g^{\mu \nu}+\frac{k^{\mu} v^{\nu}+k^{\nu} v^{\mu}}{v \cdot k}-\frac{v^{\mu} v^{\nu} k^{2}}{(v \cdot k)^{2}}\right)
\end{aligned}
$$

\footnotetext{
${ }^{4}$ This is of course nothing but a Laplace transformation with a different convention for the variable conjugate to $t$.
} 
In Eq. (44) $\Phi^{\mu}$ is an analytic function of $k^{0}$ off the real axis which depends linearly on the initial values at $t=0$ for $A(\boldsymbol{k}), \dot{A}(\boldsymbol{k})$, and $\delta \hat{f}(\boldsymbol{k}, \boldsymbol{p})$. After fixing the gauge one can solve Eq. (4) for $A(k)$. If $f(\boldsymbol{p})$ depends on the direction of $\boldsymbol{p}, A(k)$ in general has poles on the imaginary $k^{0}$-axis which yield exponentially growing solutions for $A(t, \boldsymbol{k})$.

Following Ref. [7] we introduce ${ }^{5}$

$$
m^{2} \equiv 2 N g^{2} \int \frac{d^{3} p}{(2 \pi)^{3}} \frac{f(\boldsymbol{p})}{|\boldsymbol{p}|}
$$

in order to characterize the size of $\Pi^{\mu \nu}$. The physical interpretation of $m^{2}$ depends on further properties of $\Pi^{\mu \nu}$. For example, in an isotropic plasma gluon fields with $k_{0} \sim|\boldsymbol{k}| \lesssim m$ are dynamically screened. For anisotropic plasmas there are unstable gauge field modes which grow exponentially with a growth rate of order $m$ or smaller. For a mildly anisotropic plasma only modes with $|\boldsymbol{k}| \lesssim m$ are unstable while for strongly anisotropic plasmas there are also unstable modes with $|\boldsymbol{k}| \gg m$.

In the bottom-up scenario there are contributions to the polarization tensor from different scales for $\boldsymbol{p}$. For example, in the first stage $\left(1 \ll Q \tau \ll g^{-3}\right) m^{2}$ receives contributions from hard and soft gluons which are parametrically of the same size. In this case $m^{2}$ is the sum of $m_{\mathrm{h}}^{2}$ and $m_{\mathrm{s}}^{2}$, which are defined as in Eq. (6), but with the $\boldsymbol{p}$-integration restricted to hard and soft momenta, respectively. The hard gluons are strongly anisotropic, while the soft gluons are close to being isotropic. Therefore there might be no instabilities if only the soft gluons were present. For the subsequent discussion the magnitude of the hard and soft momenta play no role. These only enter the values of $m_{\mathrm{h}}$ and $m_{\mathrm{s}}$, so we can simply think of the high momentum gluons as some two-component plasma.

Naively one might think that if one adds some isotropically distributed particles the instabilities might disappear. That this is not necessarily the case can be easily understood from the criterion for the occurrence of instabilities of Ref. [7]. It states that there is an instability associated with a given wave vector $\boldsymbol{k}$ for each negative eigenvalue of the matrix $\lim _{\epsilon \rightarrow 0}\left[\boldsymbol{k}^{2} \delta^{i j}-k^{i} k^{j}+\Pi^{i j}(i \epsilon, \boldsymbol{k})\right]$. For an isotropic distribution $\Pi^{i j}(k)$ vanishes in this limit, so adding particles with isotropic distribution does not affect the occurrence of plasma instabilities ${ }^{6}$.

We write $f=f_{\mathrm{h}}+f_{\mathrm{s}}$ and correspondingly $\Pi^{\mu \nu}=\Pi_{\mathrm{h}}^{\mu \nu}+\Pi_{\mathrm{s}}^{\mu \nu}$ and we assume that $f_{\mathrm{s}}$

\footnotetext{
${ }^{5} \operatorname{In}[7] m^{2}$ is called $m_{\infty}^{2}$, and parametrically it is the same as $m_{\mathrm{D}}^{2}$ of Ref. [5].

${ }^{6}$ It is of course possible that the added particles increase the collision rate of the gluons which contribute to $f$ to the extent that the collisionless approximation, which was used to obtain this criterion, breaks down. Then the instabilities might disappear.
} 
only depends on $|\boldsymbol{p}|$. Then $\Pi_{\mathrm{s}}^{\mu \nu}$ has exactly the same form as a polarization tensor in thermal equilibrium [20] with the Debye mass $m_{\mathrm{D}}$ replaced by $\sqrt{2} m_{\mathrm{s}}$.

We are interested in hard gluon distributions for which the typical $p_{z}$ is small compared to its transverse momentum, i.e., the typical $z$-component of $\boldsymbol{v}$ in Eqs. (15) $v_{\mathrm{h}}^{z}$ is much smaller than one. Detailed discussions of the resulting polarization tensor can be found in Refs. [7, 19]. There are no instabilities for $|\boldsymbol{k}| \gg k_{\max }$ with

$$
k_{\max } \sim \frac{m_{\mathrm{h}}}{v_{\mathrm{h}}^{z}}
$$

To keep the present discussion as simple as possible we consider $\left|\boldsymbol{k}_{\perp}\right| \ll\left|k_{0}\right|$ and $v_{\mathrm{h}}^{z}\left|k_{z}\right| \ll\left|k_{0}\right|$ so that we can approximate $v \cdot k \simeq k_{0}$ in the second line of Eq. (5). We assume that $f_{\mathrm{h}}$ is invariant under $\boldsymbol{p} \rightarrow-\boldsymbol{p}$ and under rotations around the $z$-axis. Then one finds

$$
\Pi_{\mathrm{h}}^{i j}(k) \simeq\left(\delta^{i j}-\delta^{i z} \delta^{j z}\right) \Pi_{\mathrm{h}}^{\perp}(k)
$$

with

$$
\Pi_{\mathrm{h}}^{\perp}(k)=\frac{m_{\mathrm{h}}^{2}}{2} \frac{k_{0}^{2}+\boldsymbol{k}^{2}}{k_{0}^{2}}
$$

Note that this is negative when $k_{0}=i \gamma$ and $\gamma^{2}<\boldsymbol{k}^{2}$. With the same approximations the soft contribution to $\Pi^{i j}$ can be written as

$$
\Pi_{\mathrm{s}}^{i j}(k) \simeq\left(\delta^{i j}-\delta^{i z} \delta^{j z}\right) \Pi_{\mathrm{s}}^{\perp}(k)+\delta^{i z} \delta^{j z} \Pi_{\mathrm{s}}^{z z}(k)
$$

Then there are two unstable modes with polarization approximately orthogonal to $\hat{\boldsymbol{z}}$ and their growth rate $\gamma$ is determined by

$$
\gamma^{2}+\boldsymbol{k}^{2}+\Pi^{\perp}(i \gamma, \boldsymbol{k})=0
$$

where $\Pi^{\perp}=\Pi_{\mathrm{h}}^{\perp}+\Pi_{\mathrm{s}}^{\perp}$. As we have discussed above, including $\Pi_{\mathrm{s}}^{\perp}$ in Eq. (11) does not eliminate instabilities but it decreases their growth rate because both $\Pi_{\mathrm{h}}^{\perp}(i \gamma, \boldsymbol{k})$ and $\Pi_{\mathrm{s}}^{\perp}(i \gamma, \boldsymbol{k})$ increase monotonically with increasing $\gamma$ and because $\Pi_{\mathrm{s}}^{\perp}(i \gamma, \boldsymbol{k}) \geq 0$.

First consider the case $m_{\mathrm{s}} \sim m_{\mathrm{h}}$. For $|\boldsymbol{k}| \sim m_{\mathrm{h}}$ there is only one scale in Eq. (11) and the instabilities grow with a rate of order $m_{\mathrm{h}}$. When $k_{\max } \gg|\boldsymbol{k}| \gg m_{\mathrm{h}}$, one would have $\gamma \simeq m_{\mathrm{h}} / \sqrt{2}$ if the soft gluons were absent. Since the soft gluons decrease the growth rate we have $\gamma \ll|\boldsymbol{k}|$. Then one can approximate

$$
\Pi_{\mathrm{s}}^{\perp}(k) \simeq-i \frac{\pi}{2} m_{\mathrm{s}}^{2} \frac{k_{0}}{|\boldsymbol{k}|} \quad\left(\left|k_{0}\right| \ll|\boldsymbol{k}|, \quad \operatorname{Im}\left(k^{0}\right)>0\right)
$$


Using Eqs. (92) and (12) and neglecting $\gamma^{2} \ll \boldsymbol{k}^{2}$, Eq. (11) becomes a cubic equation for $\gamma$

$$
\boldsymbol{k}^{2}-\frac{1}{2} m_{\mathrm{h}}^{2} \frac{\boldsymbol{k}^{2}}{\gamma^{2}}+\frac{\pi}{2} m_{\mathrm{s}}^{2} \frac{\gamma}{|\boldsymbol{k}|}=0
$$

Since $\gamma \ll|\boldsymbol{k}|$ the third term on the left hand side is small compared to the second and can be neglected which again gives

$$
\gamma \simeq \frac{m_{\mathrm{h}}}{\sqrt{2}} \quad\left(m_{\mathrm{h}} \sim m_{\mathrm{s}}, m_{\mathrm{h}} \ll|\boldsymbol{k}| \ll k_{\mathrm{max}}\right)
$$

i.e., in this regime the effect of soft gluons on the growth rate is negligible.

Now we consider what happens if $m_{\mathrm{s}} \gg m_{\mathrm{h}}$. We will find growth rates which are small compared to $|\boldsymbol{k}|$. Therefore we can use Eq. (13) to compute $\gamma$. First consider small $|\boldsymbol{k}|$. We neglect the first term in Eq. (13) which will be justified in a moment to obtain

$$
\gamma \simeq\left(\frac{m_{\mathrm{h}}^{2}}{\pi m_{\mathrm{s}}^{2}}\right)^{1 / 3}|\boldsymbol{k}|
$$

Now we see that one can indeed neglect the first term in Eq. (13) as long as $|\boldsymbol{k}| \ll$ $\left(m_{\mathrm{s}} / m_{\mathrm{h}}\right)^{2 / 3} m_{\mathrm{h}}$. In this range the growth rate is reduced by the soft gluons and it increases linearly with $|\boldsymbol{k}|$. Going to larger $|\boldsymbol{k}|$ all terms in Eq. (13) become of the same order of magnitude and the full cubic equation for $\gamma$ needs to be solved. Finally, when $|\boldsymbol{k}| \gg\left(m_{\mathrm{s}} / m_{\mathrm{h}}\right)^{2 / 3} m_{\mathrm{h}}$ one can neglect the last term on the left hand side of (13) which gives

$$
\gamma \simeq \frac{m_{\mathrm{h}}}{\sqrt{2}} \quad\left(m_{\mathrm{h}} \ll m_{\mathrm{s}}, m_{\mathrm{h}}^{1 / 3} m_{\mathrm{s}}^{2 / 3} \ll|\boldsymbol{k}| \ll k_{\max }\right)
$$

Thus, in this case the effect of soft gluons on the growth rate is negligible. Note that the range in which Eq. (16) holds only exists when $v_{\mathrm{h}}^{z} \ll\left(m_{\mathrm{h}} / m_{\mathrm{s}}\right)^{2 / 3}$.

\section{Plasma instabilities and bottom-up scenario}

We will now address the question whether and how plasma instabilities affect the parametric estimates of the bottom-up scenario [5]. It is clear that they have a dramatic impact if they can grow as large as in QED, i.e., if they do not saturate until $A \sim p / g$ 9]. In this case the unstable modes grow so large that they change the momenta of 
the hard particles by an amount of order one within a time of order $m^{-1}$, which would lead to a quick isotropization. We will assume that the instabilities already saturate when $A \sim k / g$, as indicated by recent lattice simulations [13, 14]. Then the effect of the instabilities on the hard gluons is much less dramatic.

We only discuss the first stage of the bottom-up scenario when the hard and the soft contributions to $\Pi^{\mu \nu}$ are of the same order of magnitude, i.e., $m_{\mathrm{s}} \sim m_{\mathrm{h}} \sim m$ and the distribution of soft gluons is nearly isotropic. We have seen in Sec. 2 that there are plasma instabilities irrespective of the numerical factors in $m_{\mathrm{s}}$ and $m_{\mathrm{h}}$.

The typical longitudinal momentum of hard gluons decreases due to the expansion. If they were free streaming, one would have $p_{\mathrm{h}}^{z} \propto \tau^{-1}$. On the other hand, $p_{\mathrm{h}}^{z}$ increases due to multiple scattering. Combining these two effects one obtains $p_{\mathrm{h}}^{z} \propto \tau^{-1 / 3}[\underline{5}]$. We will now consider how the instabilities affect this estimate.

In the linear regime the unstable modes cannot be described as particles, but rather as a coherent classical field. When its amplitude becomes of order $\mathrm{m} / \mathrm{g}$ its equation of motion becomes non-linear. Due to the complicated interaction the coherent nature of the gluon field will at least partly be lost. Parametrically the occupation number of the $|\boldsymbol{k}| \sim m$ modes is then $f_{m} \sim 1 / g^{2}$. A quantitative description of their dynamics by a Boltzmann equation is not possible because it would require that $f_{m} \ll 1 / g^{2}$. But since this is right at the border of validity of the Boltzmann equation one should still be able to use it for parametric estimates which is what we will do in the following.

Consider $2 \rightarrow 2$ scattering of a hard gluon with a $|\boldsymbol{k}| \sim m$ gluon with a momentum transfer of order $m$. The corresponding cross section $\sigma$ is of order $g^{4} / m^{2}$. Therefore the rate at which a hard gluon experiences such collisions is

$$
\frac{d N_{\mathrm{col}}}{d \tau} \sim \sigma n_{m} f_{m} \sim m
$$

where we have used $n_{m} \sim m^{3} / g^{2}$ for the number density of $|\boldsymbol{k}| \sim m$ gluons and where $f_{m}$ entered as a Bose enhancement factor. The hard gluons experience many random collisions each with a momentum transfer of order $m$ so that

$$
\left(p_{\mathrm{h}}^{z}\right)^{2} \sim N_{\mathrm{col}} m^{2} \sim \tau \frac{d N_{\mathrm{col}}}{d \tau} m^{2}
$$

In the bottom-up scenario $m^{2} \sim g^{2} n_{\mathrm{h}} / Q \sim Q^{2} /(Q \tau)$ where $n_{\mathrm{h}}$ is the number density of hard gluons. Thus these collisions lead to

$$
p_{\mathrm{h}}^{z} \sim Q(Q \tau)^{-1 / 4}
$$


which is larger than in the bottom-up scenario where $p_{\mathrm{h}}^{z} \sim Q(Q \tau)^{-1 / 3}$.

We have thus seen that plasma instabilities indeed lead to a certain isotropization in an expanding gluon plasma, even if they are saturated by non-abelian interactions. But the isotropization is far from complete, and whether the instabilities really lead to equilibration is not clear.

Let us finally use Eq. (19) to determine $k_{\max }$ of Eq. (7), i.e., the largest $|\boldsymbol{k}|$ for which there are plasma instabilities. We have

$$
v_{\mathrm{h}}^{z} \sim \frac{p_{\mathrm{h}}^{z}}{Q} \sim(Q \tau)^{-1 / 4}
$$

which gives

$$
k_{\max } \sim \frac{Q}{(Q \tau)^{1 / 2}}(Q \tau)^{1 / 4} \sim Q(Q \tau)^{-1 / 4}
$$

It is interesting that the result (21) coincides with $p_{\mathrm{h}}^{z}$ in Eq. (19).

Acknowledgments I would like to thank R. Baier, M. Laine, G. D. Moore, A. H. Mueller, P. Romatschke, K. Rummukainen, D. Schiff, A. Shoshi, and L. Yaffe for interesting discussions or comments. This work was supported in part by the DFG under grant FOR 339/2-1, and through the DFG funded Graduate School GRK 881.

\section{References}

[1] See Ref. [5] and references therein.

[2] A. H. Mueller, "Toward equilibration in the early stages after a high energy heavy ion collision," Nucl. Phys. B 572 (2000) 227 arXiv:hep-ph/9906322;; "The Boltzmann equation for gluons at early times after a heavy ion collision," Phys. Lett. B 475 (2000) 220 arXiv:hep-ph/9909388.

[3] J. Bjoraker and R. Venugopalan, "From colored glass condensate to gluon plasma: Equilibration in high energy heavy ion collisions," Phys. Rev. C 63 (2001) 024609 arXiv:hep-ph/0008294.

[4] S. M. H. Wong, "Thermal and chemical equilibration in a gluon plasma," Nucl. Phys. A 607, 442 (1996) arXiv:hep-ph/9606305. 
[5] R. Baier, A. H. Mueller, D. Schiff and D. T. Son, “'Bottom-up' thermalization in heavy ion collisions," Phys. Lett. B 502 (2001) 51 arXiv:hep-ph/0009237.

[6] S. Mrowczynski, "Plasma instability at the initial stage of ultrarelativistic heavy ion collisions," Phys. Lett. B 314 (1993) 118.

[7] P. Arnold, J. Lenaghan and G. D. Moore, "QCD plasma instabilities and bottomup thermalization," JHEP 0308 (2003) 002 arXiv:hep-ph/0307325.

[8] P. Arnold and J. Lenaghan, "The abelianization of QCD plasma instabilities," Phys. Rev. D 70 (2004) 114007 arXiv:hep-ph/0408052.

[9] P. Arnold, J. Lenaghan, G. D. Moore and L. G. Yaffe, "Apparent thermalization due to plasma instabilities in quark gluon plasma," Phys. Rev. Lett. 94 (2005) 072302 arXiv:nucl-th/0409068.

[10] U. W. Heinz, "Thermalization at RHIC," AIP Conf. Proc. 739 (2005) 163 arXiv:nucl-th/0407067.

[11] A. Rebhan, P. Romatschke and M. Strickland, "Hard-loop dynamics of non-Abelian plasma instabilities," Phys. Rev. Lett. 94 (2005) 102303 arXiv:hep-ph/0412016.

[12] A. Dumitru and Y. Nara, "QCD plasma instabilities and isotropization," Phys. Lett. B 621 (2005) 89 arXiv:hep-ph/0503121.

[13] P. Arnold, G. D. Moore and L. G. Yaffe, "The fate of non-abelian plasma instabilities in 3+1 dimensions," arXiv:hep-ph/0505212.

[14] A. Rebhan, P. Romatschke and M. Strickland, "Dynamics of quark-gluon plasma instabilities in discretized hard-loop approximation," arXiv:hep-ph/0505261.

[15] A. H. Mueller, A. I. Shoshi and S. M. H. Wong, "A possible modified 'bottom-up' thermalization in heavy ion collisions," arXiv:hep-ph/0505164.

[16] U. W. Heinz, "Kinetic Theory For Nonabelian Plasmas," Phys. Rev. Lett. 51 (1983) 351.

[17] For a review of the Vlasov equations in QED see, e.g., Ref. [18].

[18] E.M. Lifshitz, L.P. Pitaevskii, Physical Kinetics (Pergamon Press, Oxford 1981). 
[19] P. Romatschke and M. Strickland, "Collective modes of an anisotropic quark gluon plasma," Phys. Rev. D 68 (2003) 036004 arXiv:hep-ph/0304092.

[20] For a review see, e.g., U. Kraemmer and A. Rebhan, "Advances in perturbative thermal field theory," Rept. Prog. Phys. 67 (2004) 351 arXiv:hep-ph/0310337. 